\title{
Automatic Systole-Diastole Classification of Mitral Valve Complex from RT-3D Echocardiography based on Multiresolution Processing
}

\author{
Gary K. W. Tsui ${ }^{a}$, Kwan-Yee K. Wong ${ }^{a}$, Alex P. W. Lee ${ }^{b}$ \\ ${ }^{a}$ Department of Computer Science, The University of Hong Kong, Hong Kong \\ ${ }^{b}$ Division of Cardiology, Department of Medicine \& Therapeutics, The Chinese University of Hong \\ Kong, Hong Kong
}

\begin{abstract}
Mitral valve repair is one of the most prevalent operations for various mitral valve conditions. Echocardiography, being famous for its low-cost, non-invasiveness and speediness, is the dominant imaging modality used for carrying out mitral valve condition analysis in both pre-operative and intra-operative examinations. In order to perform analysis on different phases of a cardiac cycle, it is necessary to first classify the echocardiograhic data into volumes corresponding to the systole and diastole phases. This often requires tedious manual work. This paper presents a fully-automatic method for systolediastole classification of real-time three-dimensional transesophageal echocardiography (RT-3D-TEE) data. The proposed method first resamples the data with radial cutting planes, segments the mitral valve by thresholding, and removes noise by median filtering. Classification is then carried out based on the number of identified mitral valve regions. A multiresolution processing scheme is proposed to further improve the classification accuracy by aggregating classification results obtained from different image resolution scales. The proposed method was evaluated against the classification results produced by a cardiologist. Experimental results show that the proposed method, without the use of computationally intensive algorithms or the use of any training database, can achieve a classification accuracy of $91.04 \%$.
\end{abstract}

Keywords: RT-3D Echocardiography, Mitral Valve, Systole-Diastole Classification, Cardiac Cycle, Ultrasound, Multiresolution

\section{DESCRIPTION OF PURPOSE}

Lup-dub is the distinctive sound that echoes throughout our entire life. Anatomically, it is the heart-beating sound produced by two groups of valves, namely the atrioventricular valves and the semilunar valves. Beating along with the group that produces the first lup, the rhythmically repeated opening and closing of the mitral valve allows oxygenated blood to flow from the left atrium to the left ventricle. Acting as a one-way door, another vital role of the mitral valve is to prevent any backflow of blood. The mitral valve is composed of the anterior mitral leaflet and the posterior mitral leaflet. In a normal functioning mitral valve, the coaptation of the anterior and posterior mitral leaflets must be tight enough to prevent blood from flowing back into the left atrium during systole. ${ }^{1}$ Certain mitral valve conditions such as mitral regurgitation, mitral valve prolapse, or mitral stenosis, may cause inaccurate or improper opening or closing of the mitral valve during a cardiac cycle.

Mitral valve repair is a preferential choice over mitral valve replacement for various pathological conditions of the mitral valve. ${ }^{2,3}$ In mitral valve repair pre-operative procedures and intra-operative settings, two-dimensional (2D), threedimensional (3D) and real-time three-dimensional (RT-3D) transthoracic echocardiography (TTE) and transoesophageal echocardiography(TEE) are widely adopted non-invasive imaging modalities for quantifying the conditions severity, progression and repercussion. ${ }^{4-8}$ In their review, Wong et al. also suggested that cardiac surgeons should be familiar with the various echocardiographic views for making optimal assessment of annulus dilation, pinpointing the ruptured chordae, spotting perforations of a leaflet, identifying the papillary muscle dysfunction, analyzing the LV function and calcification, and determining the thickening of the subvalvular apparatus. ${ }^{9}$

Observation and assessment based on different phases of a cardiac cycle is often required. For example, patients with classic Barlow's Disease would have mitral regurgitation at mid-to-late phase of the systole, whereas patients with fibroelastic deficiency with flail leaflets would have mitral regurgitation throughout the systole. ${ }^{10}$ In order to make such investigative and objective measurements of the systolic or diastolic functions of the left atrium and left ventricle using RT-3D-TEE/TTE, much repetitive and manual work must be done. ${ }^{11,12}$ With RT-3D-TEE/TTE, part of the pre-operative

Medical Imaging 2013: Image Processing, edited by Sebastien Ourselin, David R. Haynor,

Proc. of SPIE Vol. 8669, 866946 - C 2013 SPIE - CCC code: 1605-7422/13/\$18

doi: $10.1117 / 12.2006611$

Proc. of SPIE Vol. 8669 866946-1 
echocardiographic measurements is to manually select the left ventricular end-systolic and end-diastolic volumes for other quantitative analysis of the mitral valve complex such as volume calculations, contour tracing, color-kinesis or mitral annulus identification. ${ }^{13-16}$

An automatic algorithm for rapid online analysis of the mitral valve anatomy and function is particularly useful during interventional procedures, when therapeutic decision needs to be made within minutes or even seconds. A combination of methods that searches for the correlation coefficients of the pixel intensity peaks within a manually defined region-ofinterests in each time-step from 2D echocardiograms was proposed. ${ }^{17}$ Graser et al. then proposed a method that could automatically detect a cardiac cycle on RT-3D-TEE correctly with an accuracy of $78 \% .^{18}$

In this paper, a method for automatic detection of the cardiac cycles from RT-3D-TEE images of the mitral valve is proposed. Specifically, our proposed work is able to automatically and accurately classify whether a 3D-TEE volume is in systole or diastole of a cardiac cycle.

\section{METHODS}

The end-systolic volume is defined as the last frame before the opening of the mitral valve while the end-diastolic volume is the last frame before the closure for the mitral valve. ${ }^{19}$ In the context of image processing, a set of image-slices from the systolic volumes should have a single large region of cardiac tissues (brighter pixels) while another set of image-slices from the diastolic volumes should have two large disconnected regions of cardiac tissues. As illustrated in Figure 1, our proposed method is composed of the two stages, namely i) volumetric data preprocessing and ii) multiresolution based systole-diastole classification.

\subsection{Volumetric data preprocessing}

Every set of RT-3D-TEE data consists of a number of volumes $\Omega_{k}$ each of which represents a time-step $k$ of a cardiac cycle. Each volume embodies a large number of voxels and each voxel $v_{i}$ is essentially a numerical value that indicates the intensity of the ultrasound signal in that specific spatial location $\left(x_{i}, y_{i}, z_{i}\right)$. Hence, our proposed method starts with a preprocessing stage that begins with a radial-plane cut around the center of each volume $v_{c}$. Each radial plane $p$ is evenly spaced at an angular offset $\theta$ (Figure 2(a)). Since the normal functioning mitral valve is more or less radially symmetric, the use of radial-plane cut could render a consistent view of the mitral valve complex. For ease of computation, our method defines $\theta$ as $1^{\circ}$, meaning a total of $M$, defined as 180 , symmetrically radial slices will be extracted from each volume. Next, for each radial plane, a binarization filter and a median filter are applied. The binarization filter is a good approach to separate the cardiac tissues from the blood as both masses sit at different bands of pixel intensities while the median filter is efficient at removing random noise that is inherent in the echocardiographic images.

\subsection{Multiresolution based systole-diastole classification}

In this proposed method, the objective of multiresolution processing is to identify the object-of-interests that consistently appear from coarser resolution level to finer resolution level. Here, the object-of-interests are the disconnected cardiac tissues, which also acts as an indicator for the state of the diastole. In the event of a left ventricular systole, the systolic pressure shuts the mitral valve and drives the blood through the now-opened aortic valve to the body, which can confuse our classification. To handle such a case, our proposed method applies a dilation operation to various resolution scales with a linear structural element of proportional size so that the closure of the mitral valve is preserved and at the same time the opening of the aortic valve is disregarded. The linear structural element, when applied, can morphologically minimise the opening induced by the aortic valve. At each resolution level $c$, the number of connected regions, $r_{c}$, is computed based on a 8-connected neighborhood connectivity. For this proposed method, we use 5 resolution scales at 1/10,1/5, 3/10, 2/5 and $1 / 2$ of its original size(Figure 3 and Figure 4). In order to classify whether a given volume is in diastole or systole, under this multiresolution processing scheme, the proposed method computes the mean number $\mu$ of connected regions of cardiac tissues across all resolution scales with the corresponding weights $\alpha_{c}$. For simplicity, $\alpha_{c}$ is set to 1 . Finally, our method classifies a volume as diastole only when that volume has a significant number of radial-plane image-slices with two large clusters of pixels and systole otherwise. This is expressed in (1), (2) and (3) where $\Omega_{k}$, as previously mentioned, denotes the 3D volumetric data at $k$ th time-step of a cardiac cycle and $\mu_{p}$ refers to mean number of connected regions at the $p$ th radial-plane image-slice. If the mitral valve complex is anatomically diseased such that the closure cannot be completely sealed at systolic volumes, $\delta$ is a threshold that can be increased to relax the classification contraint in formula 3. 


$$
\begin{gathered}
\mu_{k, p}=\frac{\sum_{c=1}^{n} r_{c} \alpha_{c}}{n} \\
g\left(\mu_{k, p}\right)= \begin{cases}1, & \text { if } \mu_{k, p}>=2 \\
0, & \text { otherwise }\end{cases} \\
f\left(\Omega_{k}\right) \in \begin{cases}\text { diastole, } & \text { if } \frac{\sum_{p=1}^{M} g\left(\mu_{k}, p\right)}{P}>=\delta \\
\text { systole, } & \text { otherwise }\end{cases}
\end{gathered}
$$

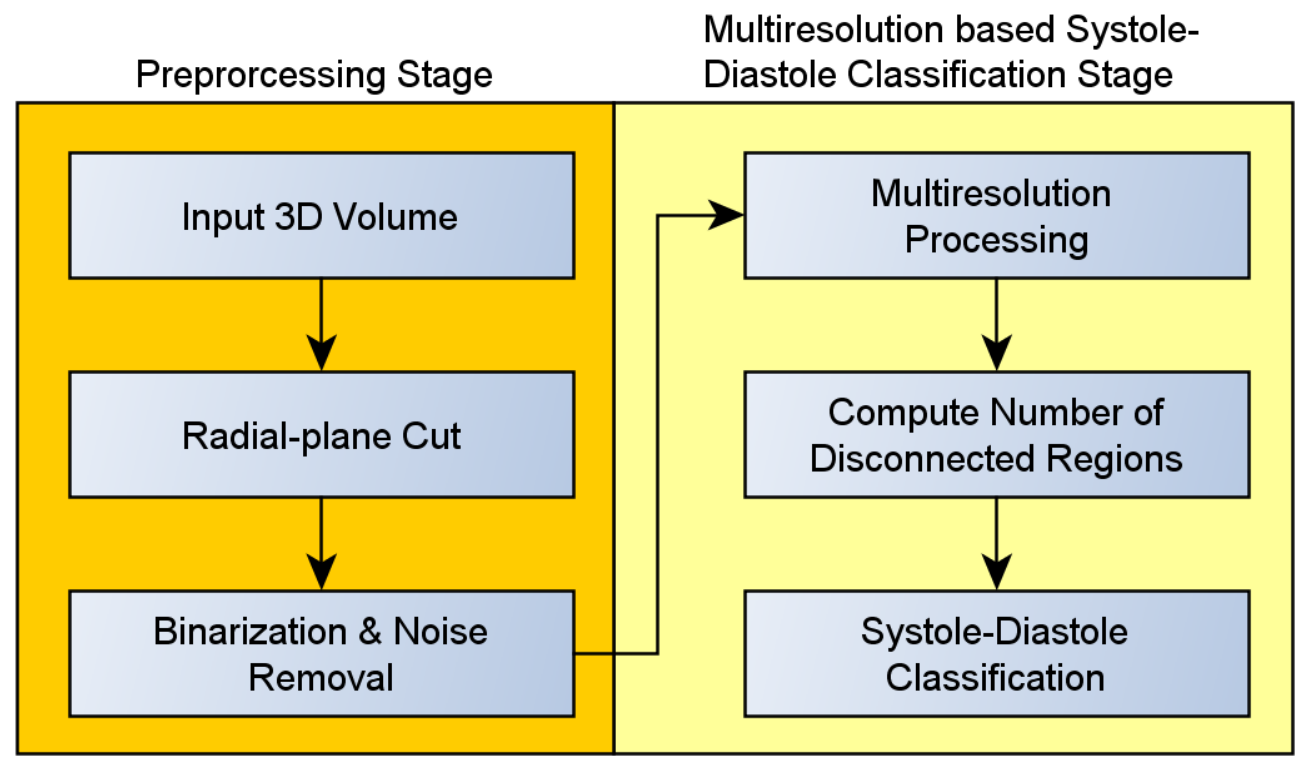

Figure 1. The flowchart of our proposed framework

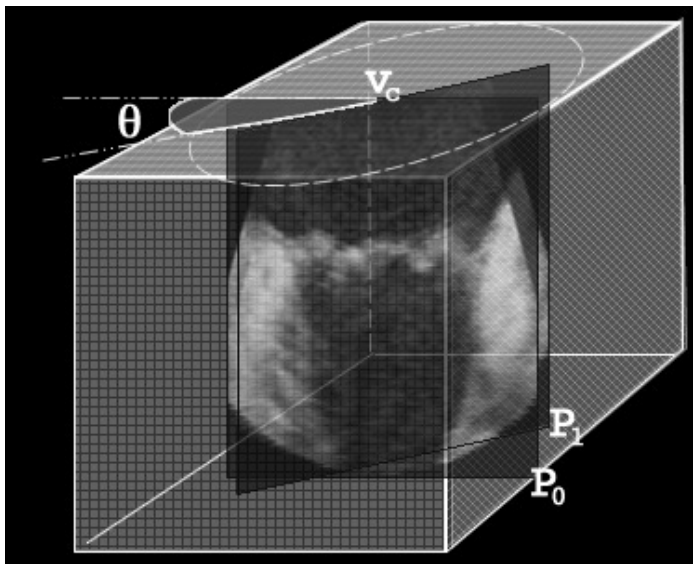

(a)

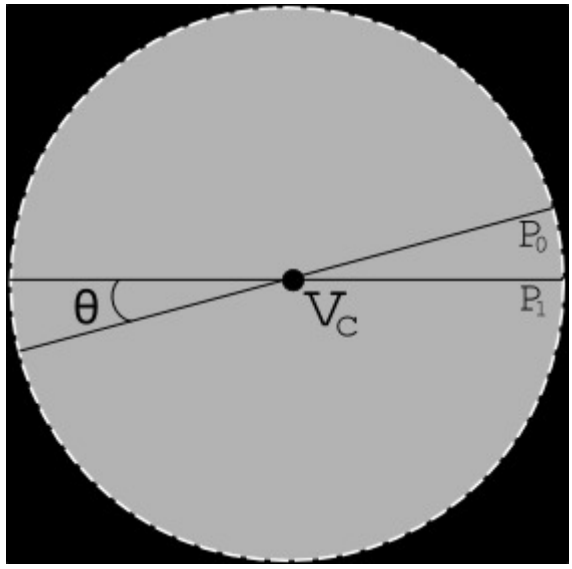

(b)

Figure 2. An example illustration of two radial-plane cuts, $P_{0}$ and $P_{1}$ with angular offset $\theta$ and center $v_{c}$, derived from the 3D echocardiographic volume of the mitral valve complex. (a) 3D illustration (b) top-view illustration 


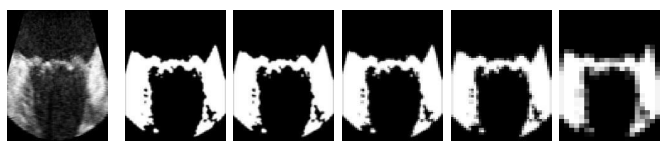

(a)

(b)

Figure 3. An example illustration of multiresolution processing. (a) Original image in systole (b) Processing under various resolution scales

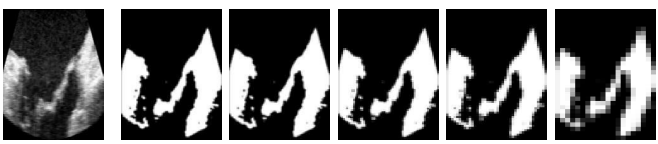

(a)

(b)

Figure 4. An example illustration of multiresolution processing. (a) Original image in diastole (b) Processing under various resolution scales

\section{RESULTS}

A total of 176 RT-3D-TEE volumes from 5 patients were processed. The dimensions of the volumes were roughly $200 \times 200 \times 200$ voxels. And, in our experiments, we have defined $\delta$ to be 0.3 as we observed that the mitral valve can have a partial opening in early-diastole phase and this setting produces reasonable results as shown below.

Our sets of clinical RT-3D-TEE data are all de-identified and are patients with a normal functioning mitral valve. The ground truth was produced by a cardiologist who manually labelled each volume (a time-step) as systole or diastole. We compares our automatic classification results against the ground truth produced by the cardiologist. Table 1 shows how we defined true positive, false positive, true negative, and false negative.

Table 1. Definition of the confusion matrix for the systole-diastole classification evaluation

\begin{tabular}{|c|c|c|c|}
\cline { 3 - 4 } \multicolumn{2}{c|}{} & \multicolumn{2}{c|}{ Predicted class } \\
\cline { 3 - 4 } \multicolumn{2}{c|}{ Actual Class } & Systole & Diastole \\
\cline { 2 - 4 } & Diastole & True Positive & False Negative \\
\hline
\end{tabular}

In order to evaluate the effect of the multiresolution processing scheme, we carried out two sets of experiments, one without and one with the multiresolution processing scheme. In the experiment without the multiresolution processing scheme, we simply used the original image scale for systole-diastole classification. Table 2 shows the result without the multiresolution scheme. The average classification accuracy is $79.42 \% \pm 10.59 \%$.

Table 3 shows the result with the multiresolution processing scheme. The average classification accuracy is $91.04 \%$ $\pm 7.84 \%$. In general, our proposed method achieved an accuracy over $92 \%$ except for valve \#4. We noticed in a few time-steps, the center of the closed mitral valve lies at an off-center location which caused our method to obtain only a partial view of that mitral valve complex after the radial-plane cut phase.

Table 2. Evaluations results for all 5 different valves(from 5 different patients) without the multiresolution processing scheme

\begin{tabular}{ccccc||c}
\hline Valve & Number of Timesteps & Precision Rate & Sensitivity(Recall Rate) & Specificity & Accuracy \\
\hline 1 & 28 & 1.00 & 0.73 & 1.00 & 0.79 \\
2 & 30 & 0.87 & 1.00 & 0.88 & 0.93 \\
3 & 20 & 0.69 & 1.00 & 0.64 & 0.80 \\
4 & 22 & 0.38 & 1.00 & 0.53 & 0.64 \\
5 & 76 & 0.94 & 0.74 & 0.93 & 0.82 \\
\hline
\end{tabular}

Table 3. Evaluations results for all 5 different valves(from 5 different patients) with the multiresolution processing scheme

\begin{tabular}{ccccc||c}
\hline Valve & Number of Timesteps & Precision Rate & Sensitivity(Recall Rate) & Specificity & Accuracy \\
\hline 1 & 28 & 1.00 & 0.88 & 1.00 & 0.93 \\
2 & 30 & 0.94 & 1.00 & 0.93 & 0.97 \\
3 & 20 & 1.00 & 0.92 & 1.00 & 0.95 \\
4 & 22 & 0.83 & 0.77 & 0.77 & 0.77 \\
5 & 76 & 1.00 & 0.86 & 1.00 & 0.93 \\
\hline
\end{tabular}




\section{CONCLUSIONS}

In this paper, we proposed a method that automatically classifies RT-3D-TEE volumes into the systole and diastole phases of the cardiac cycles. To the best of our knowledge, the proposed method is the first to make such a classification fully automatic using the mutliresolution processing approach. With the multiresolution processing scheme and only a few simple image processing operations, our proposed method has achieved an average classification accuracy of $91.04 \%$. Furthermore, we have also illustrated that without the use of computationally intensive algorithms and the need of a training database, we could still achieve an average classification accuracy of $79.42 \%$. We believe that the radial-plane cut during the preprocessing stage has helped to effectively prepare a consistent set of data for the subsequent processing stages. Aside from the accuracy, we notice that, in general, both the precision rate and the specificity are lower without the use of the multiresolution processing scheme. We believe that this is desirable as it proves that the adoption of the multiresolution processing scheme minimizes the number of incorrect classification results. We also observe that the average sensitivity for the evaluation without the multiresolution processing scheme is higher. This may be due to the fact that it is easier to identity a diastole with lower resolution scales than with only its original resolution scale. In other words, the opening between the anterior mitral leaflet and posterior mitral leaflet during diastole, after being preprocessed with a radial-plane cut and a few morphological operations, is much more pronounced at a lower resolution scale.

We find out that one of the possible root causes to the incorrect classifications is largely due to the inherently low contrasts and inconsistent distribution of the speckle noise among intra-patient and inter-patient ultrasound volumes. Nonetheless the misclassifications were only limited to a time step or two within either the diastole or systole phase. There was no misclassification of the whole systole or diastole phase. Hence, to strengthen the classification accuracy, future work could include incorporating contrast enhancement techniques ${ }^{20,21}$ and incorporate prior knowledge that restricts any inconsistent classification in the middle of the systole or the diastole phase of the cardiac cycle.

Working as an automated preprocessing tool, our proposed method can be well adapted into existing mitral valve analytical frameworks that is dependent upon the selected phases of the cardiac cycle. This can greatly and reliably reduce the amount of tedious manual work that the majority of mitral valve pre-operative and intra-operative studies has.

\section{REFERENCES}

1. OGara, P., Sugeng, L., Lang, R., Sarano, M., Hung, J., Raman, S., Fischer, G., Carabello, B., Adams, D., and Vannan, M., "The role of imaging in chronic degenerative mitral regurgitation," JACC: Cardiovascular Imaging 1, 221-237 (2 March 2008).

2. Kaiser, L. R., Kron, I. L., and Spray, T. L., [Mastery of Cardiothoracic Surgery], Lippincott Williams \& Wilkins (2007).

3. Fedak, P. W., McCarthy, P. M., and Bonow, R. O., "Evolving concepts and technologies in mitral valve repair," Circulation 117, 963-974 (2008).

4. Hung, J., Papakostas, L., Tahta, S. A., Hardy, B. G., Bollen, B. A., Duran, C. M., and Levine, R. A., "Surgery for valvular heart disease: Mechanism of recurrent ischemic mitral regurgitation after annuloplasty: Continued lv remodeling as a moving target," Circulation 110, II-85-II-90 (2004).

5. Ryan, L. P., Salgo, I. S., Gorman, R. C., and III, J. H. G., "The emerging role of three-dimensional echocardiography in mitral valve repair," Seminars in Thoracic and Cardiovascular Surgery 18, 126-134 (2 2006).

6. Adams, D. H., Anyanwu, A. C., Sugeng, L., and Lang, R. M., "Degenerative mitral valve regurgitation: Surgical echocardigrophy," Current Cardiology Reports 10, 226-232 (2008).

7. Grewal, J., Mankad, S., Freeman, W. K., Click, R. L., Suri, R. M., Abel, M. D., Oh, J. K., Pellikka, P. A., Nesbitt, G. C., Syed, I., Mulvagh, S. L., and Miller, F. A., "Real-time three-dimensional transesophageal echocardiography in the intraoperative assessment of mitral valve disease," Journal of the American Society of Echocardiography 22, 34-41 (January 2009).

8. Heyning, C. M. V. d., Magne, J., Vrints, C. J., Pierard, L., and Lancellotti, P., "The role of multi-imaging modality in primary mitral regurgitation," European Heart Journal of Cardiovascular Imaging 13, 139-151 (2012).

9. Wong, R. H., Lee, A. P., Ng, C. S., Wan, I. Y., Wan, S., and Underwood, M. J., "Mitral valve repair: Past, present, and future," Asian Cardiovascular \& Thoracic Annals 18, 586-595 (2010).

10. Anyanwu, A. C. and Adams, D. H., "Etiologic classification of degenerative mitral valve disease: Barlows disease and fibroelastic deficiency," Seminars in Thoracic and Cardiovascular Surgery 19, 90-96 (2007). 
11. Kirkpatrick, J. N., Vannan, M. A., Narula, J., and Lang, R. M., "Echocardiography in heart failure," Journal of the American College of Cardiology 50(5), 381-396 (July 2007).

12. Tamborini, G., Muratori, M., Brusoni, D., Celestse, F., Maffessanti, F., Caiani, E. G., Alamanni, F., and Pepi, M., "Is right ventricular systolic function reduced after cardiac surgery? a two- and three-dimensional echocardiographic study," European Heart Journal 10(5), 630-634 (2009).

13. Vignon, P., Mor-Avi, V., Weinert, L., Koch, R., Spencer, K. T., and Lang, R. M., "Quantitative evaluation of global and regional left ventricular diastolic function with color kinesis," Circulation 97, 1053-1061 (1998).

14. Hubka, M., Lipiecki, J., Bolson, E. L., Roy W. Martin, B. M., Maza, S. R., and Sheehan, F. H., "Three-dimensional echocardiographic measurement of left ventricular wall thickness: In vitro and in vivo validation," Journal of the American Society of Echocardiography 15(2), 129-135 (2002).

15. Jenkins, C., Bricknell, K., Hanekom, L., and Marwick, T. H., "Reproducibility and accuracy of echocardiographic measurements of left ventricular parameters using real-time three-dimensional echocardiography," Journal of the American College of Cardiology 44(4), 878-886 (2004).

16. Schneider, R. J., Perrin, D. P., Vasilyev, N. V., Marx, G. R., Nido, P. J. d., and Howe, R. D., "Mitral annulus segmentation from 3d ultrasound using graph cuts," Medical Imaging, IEEE Transactions on 29(9), 1676-1687 (2010).

17. Kachenoura, N., Delouche, A., Herment, A., Frouin, F., and Diebold, B., "Automatic detection of end systole within a sequence of left ventricular echocardiographic images using autocorrelation and mitral valve motion detection," in [Proceedings of EMBS 2007], 4504-4507 (2007).

18. Graser, B., Hien, M., Rauch, H., Meinzer, H.-P., and Heimann, T., "Automatic detection of cardiac cycle and measurement of the mitral annulus diameter in 4d tee images," in [Proceedings of SPIE], (2012).

19. Nosir, Y. F., Fioretti, P. M., Vletter, W. B., Boersma, E., Salustri, A., Postma, J. T., Reijs, A. E., Cate, F. J. T., and Roelandt, J. R., "Accurate measurement of left ventricular ejection fraction by three-dimensional echocardiography: A comparison with radionuclide angiography," Circulation 94, 460-466 (1996).

20. Zong, X., Laine, A. F., and Geiser, E. A., "Speckle reduction and contrast enhancement of echocardiograms via multiscale nonlinear processing," IEEE Transactions on Medical Imaging 17, 532-540 (August 1998).

21. Sun, Q., Hossack, J. A., Tang, J., and Action, S. T., "Speckle reducing anisotropic diffusion for 3d ultrasound images," Computerized Medical Imaging and Graphics 28, 461-470 (December 2004). 